\title{
AUFSATZ
}

\section{Muss wirklich jeder ins Europäische Parlament? Kritische Anmerkungen zur Sperrklausel-Rechtsprechung aus Karlsruhe}

\author{
Volker M. Haug
}

Mit seiner nicht unerwartet negativen Entscheidung zur Drei-Prozent-Hürde im Europawahlrecht vom 26. Februar 2014 hat der Zweite Senat des Bundesverfassungsgerichts (BVerfG) mit fünf gegen drei Stimmen seine Sperrklausel-Rechtsprechung fortgesetzt, die in mehrfacher Hinsicht rigoros und problematisch zu nennen ist: Das beginnt bei dem sehr formalen (verfassungs-)rechtlichen Maßstab unter Negierung einer europarechtlichen Überlagerung, wodurch sich das Gericht der supranationalen Dimension des Europawahlrechts weitgehend entzieht (dazu 1.). Des Weiteren wird der einzige vom Gericht anerkannte Rechtfertigungsgrund für eine Sperrklausel - die Sicherung der Funktionsfähigkeit der Volksvertretung - in seinen Voraussetzungen zu sehr verengt (dazu 2.1.) und in einer Weise geprüft, die der Bedeutung und Funktion des Europäischen Parlaments (EP) schwerlich gerecht wird (dazu 2.2.). Wenig überraschend hat das BVerfG die bereits im Urteil zur Fünf-Prozent-Hürde im Europawahlrecht entwickelten Grundsätze nun auch auf die DreiProzent-Hürde angewendet, weil es weder tatsächliche noch rechtlich erhebliche Unterschiede zur Situation zwei Jahre zuvor gesehen hat (dazu 3.). Zu diesen inhaltlichen Problemen kommt hinzu, dass das BVerfG damit auch grundlegende Kritikpunkte an seiner Arbeitsweise bestätigt und verstärkt. Dies gilt sowohl für die Inkonsequenz seiner Wahlrechtsjudikatur (dazu 4.1.) als auch für den Übergriff in den Gestaltungs- und Handlungsspielraum des Gesetzgebers (dazu 4.2.).

\section{Verfassungsrechtlicher und europarechtlicher Maßstab}

\subsection{Rechtliche Ausgangslage}

Die Schwierigkeiten des Europawahlrechts fangen bereits damit an, dass es ein einheitliches europäisches Wahlrecht für das EP im Sinne einer abschließenden Vollregelung bislang nicht gibt. Hier liegt der ungewöhnliche Fall eines Mehrebenensystems vor, bei dem die Ebene des Vertretungsorgans EU und die Ebene des dafür maßgeblichen Wahlgesetzgebers - die Mitgliedstaaten - auseinanderfallen. Dieser Befund ist auch keineswegs „EU-immanent“. Vielmehr sieht das Primärrecht in Art. 223 Abs. 1 AEUV (wie schon in Vorgängerbestimmungen $)^{1}$ eine ausdrückliche Beauftragung des EP vor, einen Entwurf für ein gesamteuropäisches Wahlrecht zu erstellen (was eine seltene Ausnahme zum sonstigen Initia-

1 Vgl. BVerfGE, Bd. 129, S. 300 (Fünf-Prozent-Hürde 2011), S. 302, Rn. 2. 
tivmonopol der Europäischen Kommission darstellt). Weil dieser Auftrag unverändert seiner Erfüllung harrt ${ }^{2}$, liegt die Zuständigkeit für das Wahlrecht zum EP noch bei den nationalen Gesetzgebern der Mitgliedstaaten - jeweils selbstverständlich nur für das „eigene“ Abgeordnetenkontingent. Die Wahl der nunmehr 96 deutschen Mitglieder des EP erfolgt demnach durch das von Bundestag und Bundesrat verabschiedete Europawahlgesetz (EuWG). ${ }^{3}$ Das bedeutet jedoch nicht, dass es keine europarechtlichen Vorgaben für das nationale Europawahlrecht gäbe. So sind in Art. 14 Abs. 3 EUV, 39 Abs. 2 EU-GRCharta die Grundsätze einer allgemeinen, unmittelbaren, freien und geheimen Wahl niedergelegt. Eine weitere Präzisierung bringt als „kleine Lösung“4 (gegenüber der von Art. 223 AEUV geforderten Vollregelung) der Direktwahlakt (DWA) ${ }^{5}$, der in Art. 1 Abs. 1, 8 Abs. 2 eine Verhältniswahl vorschreibt und in Art. 3 den Mitgliedstaaten ausdrücklich freistellt, Zugangshürden festzulegen, die die Höhe von fünf Prozent nicht überschreiten dürfen.

\subsection{Eingriff in den Gleichheitsgrundsatz}

Sowohl das deutsche als auch das europäische Recht kennen in Art. 3 GG, 20 EUGRCharta einen allgemeinen Gleichheitsgrundsatz. Gleichzeitig gibt es weder im deutschen noch im europäischen Recht eine Spezialregelung für den Grundsatz der gleichen Wahl, der für das EP verbindlich wäre. So gelten die deutschen Verfassungsbestimmungen, die die Wahlrechtsgleichheit fordern, ausdrücklich nur für die Wahlen zum Bundestag (Art. 38 Abs. 1 S. 1 GG) und zu den Volksvertretungen in den Ländern, Kreisen und Gemeinden (Art. 28 Abs.1 S. 2 GG). Auf europäischer Ebene ist die Wahlrechtsgleichheit in Art. 14 Abs. 3 EUV, 39 Abs. 2 EU-GRCharta ausgeklammert, was aber nur den - bezogen auf Wählerstimmen beziehungsweise Einwohner - degressiv proportionalen Sitzkontingenten der Mitgliedstaaten (Art. 14 Abs. 2 UAbs. 1 S. 3 EUV) geschuldet ist. ${ }^{6}$ Folglich ist sowohl beim grundgesetzlichen als auch beim europäischen Rechtsmaßstab der allgemeine Gleichbehandlungsgrundsatz für die Gleichheit bei EP-Wahlen maßgeblich. Daraus folgert das BVerfG unter Bezugnahme auf Art. 3 Abs. 1 GG und den Grundsatz der Chancengleichheit politischer Parteien gemäß Art. 21 GG - was für den europäischen Gleichheitsgrundsatz ebenso gelten muss - eine Wahlrechtsgleichheit in einem streng formalen Sinn, um

2 Was sogar schon zu einer letztlich erfolglosen Untätigkeitsklage der britischen Liberalen geführt hat, vgl. EuGH vom 10. Juni 1993 - C-41/92, I-3153; den Grund für die Nichtumsetzung dieses Auftrags haben Sachverständige vor dem BVerfG in den unterschiedlichen mitgliedstaatlichen Wahlrechtstraditionen verortet, BVerfG, Urteil vom 26. Februar $2014-2$ BvE 2/13 u.a., 2 BvR 2220/13 u.a. (Drei-Prozent-Hürde 2014), Rn. 67.

3 Siehe den instruktiven Überblick der 28 nationalen Wahlrechte zum EP unter http://www.wahlrecht.de/ausland/europa.htm (Abruf am 25. März 2014).

4 Sebastian Roßner, Verfassungswidrigkeit der Fünf-Prozent-Sperrklausel im Europawahlrecht, in: NVwZ 2012, S. 22 - 25, S. 22.

5 Beschluss und Akt zur Einführung allgemeiner unmittelbarer Wahlen der Abgeordneten des Europäischen Parlaments vom 20. September 1976 (BGBl. 1977 II, S. 733/734), zuletzt geändert durch Beschluss des Rates vom 25. Juni und 23. September 2002 (BGBl. 2003 II, S. 810; BGBl. 2004 II, S. 520).

6 Vgl. Rainer Wernsmann, Verfassungsfragen der Drei-Prozent-Sperrklausel im Europawahlrecht, in: JZ 2014, S. $23-28$, S. 24. 


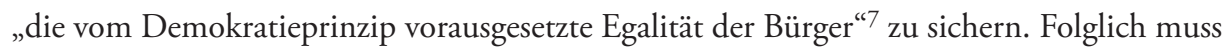
das Wahlrecht - erst recht bei einer wie vom DWA angeordneten Verhältniswahl - grundsätzlich eine Zähl- und Erfolgswertgleichheit der Wählerstimmen, also eine möglichst proportional-exakte Umsetzung der Wählerstimmen in Abgeordnetenmandate, sicherstellen. ${ }^{8}$ Es liegt auf der Hand, dass dies bei einer Sperrklausel nicht der Fall ist: Diejenigen Stimmen, die für Parteien oberhalb der faktisch nötigen Mindestzahl für einen Parlamentssitz, aber unterhalb einer Sperrklausel abgegeben wurden ${ }^{9}$, werden ihres Erfolgswertes beraubt; zugleich wachsen die damit „verfallenen“ Anteile des Proportionalsystems den Parteien mit einem Stimmenanteil oberhalb der Sperrklausel zu. Folglich gibt es Stimmen mit und Stimmen ohne Erfolgswert, was eine Ungleichbehandlung darstellt. Daher greift eine Sperrklauselregelung - und zwar unabhängig von ihrer Höhe - in dieses Gleichheitsgebot ein. ${ }^{10}$

Ein solcher Eingriff kann nur zulässig sein, wenn es dafür eine tragfähige Rechtfertigung gibt. Das BVerfG verlangt dafür Gründe, die „durch die Verfassung legitimiert und von einem Gewicht sind, das der Wahlgleichheit die Waage halten kann"11. Bis zu diesem Punkt verdient das Gericht volle Zustimmung; die Streitfrage besteht nun aber darin, ob es für die Sperrklauseln beim EP diese tragfähige Rechtfertigung gibt oder nicht.

\subsection{Rechtfertigung des Eingriffs durch eine europarechtliche Überlagerung}

\section{(a) Argumentation des BVerfG}

Aus Karlsruher Sicht ist der Fall klar: Aufgrund der ausdrücklichen Rechtssetzungsdelegation in Art. 8 Abs. 1 DWA sind die Mitgliedstaaten für das Europawahlrecht zuständig, weshalb das EuWG (natürlich) nationales Recht darstellt. Dieses unterliegt - wie jedes nationale Recht - den verfassungsrechtlichen Vorgaben (allein) des deutschen Grundgesetzes. ${ }^{12}$ Davon ausgehend verneint das Gericht eine Rechtfertigung des Eingriffs in das Wahlgleichheitsprinzip durch eine europarechtliche Überlagerung. Zwar sieht Art. 3 DWA die Möglichkeit nationaler Sperrklauseln vor. Doch handelt es sich dabei aus Karlsruher Sicht nur um eine ausdrücklich fakultativ gestellte und bei fünf Prozent gedeckelte Spielraumeröffnung für die Mitgliedstaaten, aus der sich keinesfalls eine Einengung der national-verfassungsrechtlichen Bewertung von Sperrklauseln in den Mitgliedstaaten - etwa im Sinne einer Zulässigkeitsvorgabe - ergibt. ${ }^{13}$ Die ratio legis dieser Bestimmung soll vielmehr - wie der ganze seit 2002 konkretisierte DWA - in der Beschränkung des Handlungsspielraums der nationalen Wahlrechtsgesetzgeber liegen. Jedenfalls soll keine Sperrklausel über fünf Prozent festgesetzt werden können. Für dieses Auslegungsergebnis zieht das Gericht sowohl

7 BVerfGE, Bd. 129, S. 300, S. 317, Rn. 76 - 78; BVerfG, a.a.O. (Fn. 2), Rn. 46 f.

8 Vgl. Rudolf Wendt, Sperrklauseln im Wahlrecht?, in: Matthias Ruffert (Hrsg.), Dynamik und Nachhaltigkeit des Öffentlichen Rechts: Festschrift für Professor Dr. Meinhard Schröder zum 70. Geburtstag, Berlin 2012, S. 431 - 456, S. 432 f.

92009 waren dies nach den Feststellungen des Gerichts sieben Parteien beziehungsweise circa zehn Prozent der abgegebenen gültigen Stimmen, vgl. BVerfGE, Bd. 129, S. 300, S. 319 f., Rn. 83 f.

10 BVerfGE, Bd. 129, S. 300, S. 319, Rn. 83; BVerfG, a.a.O. (Fn. 2), Rn. 51.

11 BVerfGE, Bd. 129, S. 300, S. 320, Rn. 87; BVerfG, a.a.O. (Fn. 2), Rn. 53.

12 BVerfGE, Bd. 129, S. 300, S. 317, Rn. 77; BVerfG, a.a.O. (Fn. 2), Rn. 40.

13 BVerfGE, Bd. 129, S. 300, S. 317, Rn. 77; BVerfG, a.a.O. (Fn. 2), Rn. 41. 
den Wortlaut („können“) als auch die Entstehungsgeschichte heran. Aus diesem Grund stellt sich die Frage eines Konflikts zwischen nationalem Verfassungsrecht und supranationalem Europarecht gar nicht, weshalb auch eine Vorlagepflicht des BVerfG an den EuGH „offenkundig nicht im Raum“ steht. ${ }^{14}$

\section{(b) Kritik: Verkennung der europäischen Dimension}

Der Ansatz des Senats, das EuWG aufgrund seines formalen Charakters als deutsches Bundesrecht strikt und ausschließlich an den Vorgaben des GG zu messen, mag allenfalls auf den ersten Blick schlüssig erscheinen. Denn der telos dieses Gesetzes besteht nicht darin, eine isolierte Wahl von 96 Mitgliedern eines deutschen Abgeordnetenkontingents ohne jeden Kontext zu gewährleisten, sondern den deutschen Beitrag zur gesamthänderischen Aufgabe aller Mitgliedstaaten zu leisten, die erforderlichen Rechtsgrundlagen für die Wahl des EP zu schaffen. In Verbindung mit dem Gebot der Europafreundlichkeit des GG gemäß Art. 23 GG ergibt sich daraus, dass das deutsche Verfassungsgericht beim Prüfungsmaßstab für Regelungen des EuWG stets das System und die Wertungen des supranationalen Rechts mitdenken und -berücksichtigen muss. ${ }^{15} \mathrm{Da}$ das supranationale Europarecht gleichzeitig einen Art. 3 GG entsprechenden allgemeinen Gleichheitsgrundsatz in Art. 20 EUGRCharta und die Zulässigkeit einer Sperrklausel bis zur Höhe von fünf Prozent in Art. 3 DWA kennt, hält es diese beiden Bestimmungen offensichtlich für miteinander vereinbar. Daran ändern weder die Betonung der Fünf-Prozent-Deckelung in der Entstehungsgeschichte von Art. 3 DWA noch der auf eine Ermächtigung ausgerichtete Sprachgebrauch der Vorschrift etwas. Aus diesem Wertungszusammenhang des Europarechts wäre nur ein Entkommen möglich, wenn man die Primärrechtswidrigkeit von Art. 3 DWA behaupten wolle. Da für eine solche Entscheidung aber allein der EuGH zuständig sein kann, ist dieser argumentative Weg einem nationalen Verfassungsgericht verschlossen, das eine Vorlage an den EuGH vermeiden möchte. ${ }^{16}$ Dann aber muss dieser Wertungszusammenhang von Art. 20 EU-GRCharta und Art. 3 DWA aufgrund des nicht mitgliedstaatlich beschränkten telos auch bei der verfassungsrechtlichen Prüfung von Sperrklauselregeln im EuWG berücksichtigt werden. ${ }^{17}$ Die Negierung dieser europarechtlichen Überformung der deut-

14 BVerfG, a.a.O. (Fn. 2), Rn. $41-44$.

15 Christoph Schönberger, Das Bundesverfassungsgericht und die Fünf-Prozent-Klausel bei der Wahl zum Europäischen Parlament, in: JZ 2012, S. 80 - 86, S. 81, spricht in diesem Zusammenhang von einem "Rahmen und Interpretationshorizont des Unionsrechts".

16 Auch der in der Literatur von Oliver W. Lembcke / Enrico Peuker / Dennis Seifarth, Wandel der Wahlrechtsrealitäten - Zur Verfassungswidrigkeit des $\$ 2$ Abs. 7 EuWG, in: DVBl. 2012, S. 401 - 407, S. 403, angebotene Ausweg, den Wahlrechts-Gleichheitssatz deutscher (also strengerer) Prägung zur Verfassungsidentität des GG zu zählen, überzeugt wenig, denn dafür müsste man dieses Wahlrechtsprinzip unmittelbar im Demokratieprinzip verankern (weil weder Art. 3 GG noch Art. 38 Abs. 1 GG in Art. 79 Abs. 3 GG genannt sind), das auf europarechtlicher Ebene in Art. 2, 10 EUV genauso existiert. Dann aber würde sich Deutschland nicht nur anmaßen, ein anderes - also besseres - Demokratieverständnis zu haben als die EU und die vielen anderen Mitgliedstaaten mit einer EP-Sperrklausel, sondern auch seiner eigenen Verfassung ein gespaltenes Verständnis des Demokratiebegriffs zwischen Art. 23 Abs. 1 GG und Art. 20 Abs. 1 GG unterstellen.

17 Im Ergebnis ebenso Christoph Schönberger, a.a.O. (Fn. 15), S. 81 f.; Rainer Wernsmann, a.a.O. (Fn. 6), S. 24. 
schen EP-Sperrklausel-Problematik durch das BVerfG ist somit nicht nur vor dem Hintergrund des Gebots der Europafreundlichkeit des Art. 23 GG äußerst bedenklich, sondern zeugt außerdem von einer in EU-bezogenen Rechtsfragen problematischen national geprägten Sichtweise des Senats.

\section{Rechtfertigungsgrund der „Sicherung der Funktionsfähigkeit der Volksvertretung“}

Einen unstreitig - auch vom BVerfG - anerkannten Rechtfertigungsgrund für den Eingriff in die Wahlrechtsgleichheit bildet „die Sicherung des Charakters der Wahl als eines Integrationsvorgangs bei der politischen Willensbildung des Volkes und, damit zusammenhängend, die Sicherung der Funktionsfähigkeit der zu wählenden Volksvertretung "18. Welche Anforderungen aber im Einzelnen an das Vorliegen dieses Rechtfertigungsgrundes zu stellen sind, ist streitig.

\subsection{Anforderungshöhe}

\section{(a) Argumentation des BVerfG}

Das BVerfG lässt für die Rechtfertigung des Eingriffs in die Wahlrechtsgleichheit nicht mehr die Grunderkenntnis ausreichen, dass die generelle Gefahr einer Parteien- und Parlamentszersplitterung zu den strukturellen Nachteilen eines Verhältniswahlrechts gehört. ${ }^{19}$ Vielmehr verlangt es in seiner jüngeren Sperrklausel-Rechtsprechung, die mit dem Urteil zur Fünf-Prozent-Hürde im schleswig-holsteinischen Kommunalwahlrecht 2008 ihren Anfang genommen hat ${ }^{20}$, dass die von einer Zersplitterung ausgehende Funktionsstörung nicht nur „rein theoretisch“ festgestellt wird, sondern in Orientierung an der „politischen Wirklichkeit“ „mit einiger Wahrscheinlichkeit“ zu erwarten ist. In diesem Sinne erlegt das Gericht dem Gesetzgeber eine Darlegungs- und beinahe schon Beweislast dafür auf, dass es diese tatsachengestützte Wahrscheinlichkeit gibt. ${ }^{21}$ Besonders deutlich wird dies bei der Entscheidung zur Drei-Prozent-Hürde, in der das Gericht eine Reihe von Argumenten des Gesetzgebers durch Hinweis auf denkbare Alternativkonstellationen als nicht konkret oder wahrscheinlich genug zurückweist. ${ }^{22}$ Insbesondere reicht dem Senat eine bloße Erschwerung von Mehrheitsbildungen eines Parlaments nicht aus; vielmehr muss eine „Tendenz zu einer Blockade parlamentarischer Willensbildung “ erkennbar sein. ${ }^{23}$ Zudem ist die Frage der Funktionsfähigkeit „nach den konkreten Funktionen des zu wählenden Organs“ zu bestimmen, weshalb das Gericht in der Entscheidung zur Fünf-Prozent-Hürde akribisch die Stellung des EP innerhalb des europäischen Institutionengefüges analysiert (und für unzureichend erklärt, dazu nachfolgend unter 2.2.).

18 BVerfGE, Bd. 129, S. 300, S. 320 f., Rn. 88; BVerfG, a.a.O. (Fn. 2), Rn. 54.

19 Anders als in früheren Entscheidungen, vgl. BVerfGE, Bd. 1, S. 208, S. 248 f.; BVerfGE, Bd. 51, S. 222 (Fünf-Prozent-Hürde 1979), S. $236 \mathrm{f}$.

20 BVerfGE, Bd. 120, S. 82, S. 113 ff., insbesondere Rn. 124 f.

21 BVerfGE, Bd. 129, S. 300, S. 323, Rn. 92; BVerfG, a.a.O. (Fn. 2), Rn. 54 f., 60.

22 BVerfG, a.a.O. (Fn. 2), Rn. $74 \mathrm{ff}$.

23 BVerfGE, Bd. 129, S. 300, S. 332 f., Rn. 112. 
(b) Erster Kritikpunkt: Verkennung der Bedeutung einer Verhältniswahl

Die Argumentation des BVerfG ist von einer Überhöhung der Gleichheit gegenüber anderen Prinzipien geprägt. ${ }^{24}$ Dies wird in seiner Einseitigkeit weder dem Gleichheitsprinzip selbst, noch der Funktion einer Wahl oder dem konkreten Wahlsystem der Verhältniswahl gerecht.

So bedarf zunächst der Eingriff in das Gleichheitsprinzip einer Einordnung: Es geht bei einer Sperrklausel weder darum, dass die Stimme eines einzelnen Wählers unterschiedlich stark gewichtet wird (Zählwert), noch darum, dass die Hürden für die verschiedenen politischen Wettbewerber unterschiedlich hoch sind. Anders als etwa im Kommunalwahlrecht, das den bereits im Gemeinderat vertretenen Parteien im Gegensatz zu „Newcomern“ die Unterstützungsunterschriften beim Wahlvorschlag erlässt ${ }^{25}$, muss hier jede Partei bei jeder Wahl die Sperrklausel in gleicher Weise (erneut) überwinden; dass dies manchen Parteien leichter gelingt als anderen, liegt nicht am Wahlrecht, sondern an deren stärkerem Rückhalt in der Bevölkerung. Folglich ist hier das formale Egalitätsgebot gewahrt, weil „jeder Wähler dieselbe Chance hat, mit seiner Stimme Kandidaten einer Partei zum Einzug in das Parlament unter Überwindung der Fünf-Prozent-Hürde zu verhelfen“; somit liegt nur eine „Erfolgswertdifferenzierung" vor, an deren Rechtfertigung auch nur entsprechend maßvolle Anforderungen zu stellen sind. ${ }^{26}$

Des Weiteren dient eine Wahl - auch eine Verhältniswahl - keineswegs ausschließlich der (möglichst exakten) Abbildung der im Volk vorhandenen politischen Präferenzen, sondern ebenso der Integration des politischen Willens des Volkes durch Kreation eines handlungs- und funktionsfähigen Organs. ${ }^{27}$ Gerade wegen dieser Funktion ist nach ständiger Rechtsprechung des BVerfG sogar ein Mehrheitswahlsystem unter dem GG denkbar, obwohl hier noch wesentlich mehr Stimmen (eventuell sogar deren Mehrheit) um ihren Erfolgswert gebracht werden. Diesem - auch im Sondervotum zum Urteil zur Fünf-Prozent-Hürde vorgetragenen ${ }^{28}$ - Argument ist in der Literatur vielfach entgegengehalten worden, dass man wegen des Gebots der Systemrelativität kein Problem des Verhältniswahlrechts mit einer wahlsystemfremden Erwägung des Mehrheitswahlrechts rechtfertigen könne. ${ }^{29}$ Dieser Einwand trifft vordergründig zu, verkennt jedoch, dass es hier um etwas anderes geht: Jedes Wahlsystem muss sich an den generellen - systemunabhängigen - Anforderungen eines Wahlrechts messen lassen. Hierzu gehört bei einem Organ, das wie ein

24 In der Presse ist folgerichtig von einem „übertriebenen Gleichheitspurismus“ die Rede, vgl. Günter Nonnenmacher, Was Sache der Karlsruher Richter ist, in: FAZ vom 27. Februar 2014, S. 1.

25 Siehe zum Beispiel $\$ 8$ Abs. 1, S. 3 KomWG BW.

26 Udo Di Fabio / Rudolf Mellinghoff, Abweichende Meinung, in: BVerfGE, Bd. 129, S. 300, S. 348, Rn. 150; ebenso Jörg Geerlings / Andreas Hamacher, Der Wegfall der Fünf-Prozent-Klausel bei Europawahlen, in: DÖV 2012, S. 671 - 678, S. 675.

27 Vgl. Peter Müller, Abweichende Meinung, in: BVerfG, Urteil vom 26. Februar $2014-2$ BvE 2/13 u.a., 2 BvR 2220/13 u.a., Rn. 25; Rudolf Wendt, a.a.O. (Fn. 8), S. 437; so auch noch völlig zutreffend BVerfGE, Bd. 51, S. 222, S. 236.

28 Vgl. Udo Di Fabio / Rudolf Mellinghoff, a.a.O. (Fn. 26), S. 348, Rn. 150.

29 Vgl. Oliver W. Lembcke / Enrico Peuker / Dennis Seifarth, a.a.O. (Fn. 16), S. 402; Martin Morlok, Chancengleichheit ernstgenommen - Die Entscheidung des Bundesverfassungsgerichts zur FünfProzent-Klausel bei der Europawahl, in: JZ 2012, S. 76 - 80, S. 79; Dirk Ehlers, Sicherung der Funktionsfähigkeit des Europäischen Parlaments mittels einer Sperrklausel im deutschen Wahlrecht, in: ZG 2012, S. 188 - 198, S. 196. 
Parlament einerseits auf Meinungskampf und Pluralismus sowie andererseits in seiner Willensbildungs- und Entscheidungsfähigkeit auf das Mehrheitsprinzip ausgerichtet ist, auch die Notwendigkeit, diese prägenden Organmerkmale sicherzustellen. So werden Meinungskampf und Pluralismus durch entsprechende Rede-, Antrags- und Minderheitsrechte ebenso gewährleistet wie die Willensbildungs- und Entscheidungsfähigkeit - gerade bei streitigen, komplexen und multipolaren Fragen - durch das Wahlrecht und die Geschäftsordnung. ${ }^{30}$ Diese funktionsbedingte Aufgabe kann das Wahlrecht nun - je nach System - auf verschiedene Weise erfüllen. Das Mehrheitswahlrecht leistet dies durch seine systemimmanente Begünstigung klarer Mehrheiten. Das insofern aber sehr viel problematischere Verhältniswahlrecht (mit vielen Vorzügen auf anderen Feldern) kann diese Aufgabe nur mit ergänzenden Regelungen bewältigen. Hierfür kommt (wie es der deutschen Wahlrechtstradition entspricht) eine in ihrer Höhe angemessene Sperrklausel in Betracht; andere (ebenfalls demokratische) europäische Staaten überwinden dieses Defizit des Verhältniswahlrechts mit einer Mehrheits-,„Verstärkung“ durch Bonusmandate für die Partei oder das Parteienbündnis, das die relative Mehrheit errungen hat (so genanntes „verstärktes Verhältniswahlrecht“). ${ }^{31}$ Daraus folgt, dass der mit einer maßvollen Sperrklausel verbundene Eingriff in eine angemessen gewichtete Wahlrechtsgleichheit in einem Verhältniswahlrecht durch die funktionsspezifische Zielsetzung eines jeden Wahlrechts, ein handlungsfähiges - das heißt entscheidungsfähiges - Parlament hervorzubringen, gerechtfertigt ist. ${ }^{32}$

\section{(c) Zweiter Kritikpunkt: Unzulässige Vermengung der Funktionsfähigkeit von Parlament und EU}

Ein weiterer Einwand zu den Anforderungen an die Eingriffsrechtfertigung betrifft den Bezugspunkt der Funktionsbeeinträchtigung. So bezieht sich ein wesentlicher Teil der Karlsruher Argumentation - vor allem im Urteil zur Fünf-Prozent-Hürde - gar nicht darauf, ob oder wie das Parlament „für sich genommen“ funktioniert, sondern vielmehr darauf, wie wichtig oder relevant die Handlungsfähigkeit des EP für das europäische Institutionengefüge - etwa bei der Gesetzgebung, Haushaltsverabschiedung oder Wahl der Kommission - ist. Dann aber geht es nicht um die Funktionsfähigkeit des Parlaments, sondern der EU insgesamt. Zwar ist dieser Bezugspunkt bereits in der klassischen Sperrklausel-Rechtfertigung für den Bundestag und die Landtage angelegt, wo stets auf die Bildung und kontinuierliche Unterstützung einer Regierung abgestellt wird. ${ }^{33}$ Relevant kann aber immer nur die Frage sein, ob die durch das in Rede stehende Wahlrecht zu schaffende Volksvertretung in ihrer Funktionsfähigkeit beeinträchtigt ist. ${ }^{34}$ Auf die Stellung im Institutionengefüge kann es hingegen allenfalls nachrangig ankommen. Sonst würde man ein Wahlrecht billigen müssen, das ein zwar unwichtiges, aber eben auch funktionsunfähiges

30 Vgl. Jörg Geerlings / Andreas Hamacher, a.a.O. (Fn. 26), S. 672.

31 Beispielsweise in Italien und Griechenland, vgl. Roland Kulke, Die Wahlverfahren in den Ländern Europas, http://www.europa-links.eu/fakten/die-wahlverfahren-in-den-landern-europas-199/ (Abruf am 26. März 2014).

32 Vgl. Udo Di Fabio / Rudolf Mellinghoff, a.a.O. (Fn. 26), S. 348, Rn. 151, bezeichnen die Sperrklausel in diesem Zusammenhang gar als „Annexbedingung“ des Verhältniswahlrechts.

33 Vgl. BVerfGE, Bd. 1, S. 208, S. 248; BVerfGE, Bd. 129, S. 300, S. 335, Rn. 118.

34 Vgl. RudolfWendt, a.a.O. (Fn. 8), S. 447 f.; Peter Müller, a.a.O. (Fn. 27), Rn. 26. 
Organ hervorbringt. Es liegt aber auf der Hand, dass auch dieses Wahlrecht seiner Aufgabe nicht gerecht würde.

\subsection{Anwendung der Maßstäbe auf das EP}

\section{(a) Argumentation des BVerfG}

Bei seiner Verneinung einer hinreichenden Funktionsbeeinträchtigung stützt sich das Gericht auf zwei Argumentationsstränge.

So sieht es - zum ersten - die Arbeitsfähigkeit des EP als nicht „mit der erforderlichen Wahrscheinlichkeit beeinträchtigt" an. Bei der Wahl 2009 sind 162 Parteien in das EP eingezogen; hätte es die Sperrklausel (in Deutschland) nicht gegeben, wären es 169 Parteien gewesen. Dieser geringe Zuwachs - in der Literatur auch als „quantité négligeable“ 35 bezeichnet - mag die Mehrheitsbildung zwar erschweren, aber nicht zu einer ernsthaften Funktionsbeeinträchtigung des EP führen. ${ }^{36}$ Hinzu kommt, dass die sieben Fraktionen des EP eine besondere Integrationsleistung über Sprach-, Landes-, Kultur- und Parteigrenzen hinweg erbracht haben und daher auch Anschlussmöglichkeiten für deutsche Kleinparteien bieten könnten. ${ }^{37}$

Der zweite Argumentationsansatz betrifft die Inter-Organ-Stellung des EP. Bei diesem besteht aus Karlsruher Sicht keine mit dem Bundestag oder den Landtagen vergleichbare Interessenlage, die auf Regierungsstabilität ausgerichtet wäre ${ }^{38}$, denn dem EP kommt zunächst weder eine regierungsbildende noch eine regierungstragende Funktion zu, obwohl der Kommissionspräsident auf Vorschlag des Europäischen Rates durch das EP gewählt wird (Art. 17 Abs. 7 UAbs. 1 S. 2 EUV) und die Kommission als Gesamtgremium der Zustimmung des EP bedarf (Art. 17 Abs. 7 UAbs. 3 EUV); und für ihre Amtsführung benötigt die Kommission, einmal ins Amt gelangt, nicht mehr die fortlaufende Unterstützung des EP. ${ }^{39}$ Insbesondere ermöglicht Art. 294 AEUV die Verabschiedung von Rechtsakten im Zusammenwirken von Kommission und Rat, wenn das Parlament keine positive oder negative Mehrheit zustande bringt. Daraus folgert der Senat, dass das ordentliche Gesetzgebungsverfahren „keine mehrheitsgetragene Zustimmung des Parlaments“ benötigt. ${ }^{40}$ Ähnlich verhält es sich bei der Erstellung des Haushaltsplans, der ebenfalls letztlich ohne Segen des EP verabschiedet werden kann (Art. 314 AEUV). ${ }^{41}$

35 Oliver W. Lembcke / Enrico Peuker / Dennis Seifarth, a.a.O. (Fn. 16), S. 407.

36 Vgl. BVerfGE, Bd. 129, S. 300, S. 330, S. 334 f., Rn. 107 f., Rn. 116 f.; zustimmend Martin Morlok, a.a.O. (Fn. 29), S. 78; Sebastian Roßner, a.a.O. (Fn. 4), S. 24.

37 Vgl. BVerfGE, Bd. 129, S. 300, S. 325, S. 327 ff., Rn. 98, Rn. $102-105$.

38 Noch weitergehender Oliver W. Lembcke / Enrico Peuker / Dennis Seifarth, a.a.O. (Fn. 16), S. 405 f., die dem EP die Erfüllung der klassischen Parlamentsfunktionen - vor allem die Kreations- und Kontrollfunktion - absprechen; ganz anders noch BVerfGE, Bd. 51, S. 222, S. 247: „Das Europäische Parlament ist die Vertretung der Völker der in den Gemeinschaften zusammengeschlossenen Staaten. Es hat als Gegenspieler der von den Regierungen der Mitgliedstaaten berufenen Kommission deren weitverzweigte Tätigkeiten zu kontrollieren."

39 Vgl. BVerfGE, Bd. 129, S. 300, S. 336, Rn. 118 f.; zustimmend Martin Morlok, a.a.O. (Fn. 29), S. 78.

40 BVerfGE, Bd. 129, S. 300, S. 337 f., Rn. 120 ff.; ähnlich Dirk Ehlers, a.a.O. (Fn. 29), S. 197.

41 Vgl. BVerfGE, Bd. 129, S. 300, S. 338 f., Rn. 123. 
(b) Erster Kritikpunkt: Verkennung des supranationalen Charakters und der Arbeitsweise des EP

Zunächst ist auch an diesem Punkt dem BVerfG vorzuhalten, dass seine Betrachtung der EP-Sperrklauselproblematik von einer isoliert deutschen Sichtweise geprägt ist. Natürlich sind 169 Parteien gegenüber 162 nur eine unwesentliche Erhöhung. Entscheidend ist aber wiederum, dass es sich bei der Wahl des deutschen Abgeordnetenkontingents nur um die „Teilerfüllung“ einer gesamthänderischen Aufgabe der EU-Staatengemeinschaft handelt. Wegen der damit verbundenen gemeinschaftsbezogenen Mitverantwortung muss jeder Mitgliedstaat seine Regelungen so gestalten, dass sie auch bei gleichlautender Übernahme in allen anderen Mitgliedstaaten zu einem arbeits- und funktionsfähigen Parlament führen. ${ }^{42}$ Dieser Teilverpflichtung wird Deutschland nun nicht gerecht, denn nahezu alle EUMitgliedstaaten verfügen entweder (wegen nur weniger überhaupt zu vergebender Sitze) über faktische Sperrklauseln (oft im zweistelligen Bereich) oder aber über formelle Sperrklauseln meist von vier oder fünf Prozent. Zu den wenigen Ausnahmen gehören Spanien und Großbritannien - und nun auch Deutschland. ${ }^{43}$ Würden alle übrigen EU-Staaten ebenfalls auf ihre Sperrklauseln verzichten, würden in das EP wesentlich mehr als nur sieben Splitterparteien - nämlich womöglich 40 bis 80 Kleinstparteien - zusätzlich einziehen. ${ }^{44}$

Des Weiteren überschätzt und überfordert das BVerfG die Integrationsfähigkeit und -bereitschaft der Fraktionen des EP. So ist die Integrationsfähigkeit in allererster Linie auf die aus den verschiedenen Mitgliedstaaten stammenden Parteien ähnlicher Grundüberzeugung gerichtet, was bereits eine besondere interkulturelle und politische Leistung darstellt. ${ }^{45}$ Ein Blick auf die Zusammensetzung der Fraktionen erhellt, dass nur in wenigen Ausnahmefällen Vertreter verschiedener Parteien aus demselben Mitgliedstaat in derselben Fraktion sind. Hinzu kommt, dass das BVerfG hier von den EP-Fraktionen etwas verlangt, was in Deutschland sogar verboten ist: Nach $₫ 10$ Abs. 1 S. 1 GO BT dürfen sich im Bundestag nur Abgeordnete verschiedener Parteien zu einer Fraktion zusammenschließen, wenn sie in keinem Bundesland miteinander im Wettbewerb stehen. Vor diesem Hintergrund ist es wenig parlamentarisch und politisch gedacht, dass Parteien im Wahlkampf (auf nationaler Ebene) miteinander konkurrieren und anschließend im Parlament in eine gemeinsame Fraktion eintreten sollen. Völlig glaubwürdig haben daher Abgeordnete des EP dem Gericht erklärt, dass es die dort unterstellte Aufnahmebereitschaft der Fraktionen nicht gibt. ${ }^{46}$ Ausgehend von diesem Befund wird durch die Karlsruher Rechtsprechung der Anteil nicht integrierter Abgeordneter zunehmen, was den prozentualen Anteil des politisch organisierten Parlaments und damit seine Funktionsfähigkeit nicht nur graduell mindert. Doch das BVerfG will erst bei erkennbaren Blockadetendenzen die Eingriffsrecht-

42 Vgl. Udo Di Fabio / Rudolf Mellinghoff, a.a.O. (Fn. 26), Rn. 157, Rn. 160; Peter Müller, a.a.O. (Fn. 27), Rn. 13.

43 Siehe die Zusammenstellung der Sperrklauseln aller EU-Länder unter http://www.tagesschau.de/ inland/europawahl402.html (Abruf am 28. März 2014); BVerfG, a.a.O. (Fn. 2), Rn. 23.

44 Vgl. BVerfG, a.a.O. (Fn. 2), Rn. 24.

45 Vgl. Jörg Geerlings / Andreas Hamacher, a.a.O. (Fn. 26), S. 676; Christoph Schönberger, a.a.O. (Fn. 15), S. 84.

46 Vgl. Peter Müller, a.a.O. (Fn. 27), Rn. 20; zutreffend fragt Christoph Schönberger, a.a.O. (Fn. 15), S. 84, welcher Fraktion sich der deutsche „Graue Panther" oder „Pirat" anschließen soll. 
fertigung bejahen und schraubt damit die zur Rechtfertigung notwendige Funktionsbeeinträchtigung bis an die Grenze der Funktionsunfähigkeit hinauf. Das wird weder dem Begriff einer Funktionsbeeinträchtigung, noch dem Parlament, noch dem einzelnen Wähler eines solchen Parlaments und auch nicht dem GG als Prüfungsmaßstab gerecht. ${ }^{47}$

\section{(c) Zweiter Kritikpunkt: Verkennung der Bedeutung und Stellung des EP}

Die unterschiedslose Übertragung der zum schleswig-holsteinischen Kommunalwahlrecht entwickelten Sperrklausel-Rechtsprechung auf das EP bewirkt letztlich eine fragwürdige Gleichordnung der einzigen gemeinsam unmittelbar gewählten Volksvertretung für rund 500 Millionen Menschen eines Kontinents mit dem Rat jeder x-beliebigen Gemeinde, ohne sich damit auseinanderzusetzen, welche wesensmäßigen Unterschiede zwischen einem auf administrative Aufgaben fokussierten und unter Rechtsaufsicht stehenden Verwaltungsgremium mit nur stark eingeschränkten Entscheidungs- und Gestaltungsbefugnissen und einem supranationalen Vielvölkerparlament bestehen. ${ }^{48}$ In der Literatur ist gar das Argument zu finden, dass der hürdenlose Einzug in das EP für kleine Parteien die Chance bietet, sich zu profilieren und zu etablieren, um „so mittelfristig auch den Einzug in die deutschen Landtage und den Bundestag zu schaffen“ ${ }^{49}$. Eine derartige Einordnung als „Vorstufe" für deutsche Parlamente insbesondere auf regionaler Ebene wird der Bedeutung des EP in keiner Weise gerecht.

Zwar trifft es zu, dass das EP noch Demokratie- und Bedeutungsdefizite aufweist, so etwa die ungleiche Sitzkontingentierung, das fehlende Gesetzesinitiativrecht und die - vom Gericht dargestellten - Mitwirkungsschwächen im Gesetzgebungsverfahren (weshalb man das EP als „Parlament eigener Art“ bezeichnen kann). ${ }^{50}$ Dennoch verkennt das BVerfG heute den Charakter des EP als das zentrale demokratische Legitimationszentrum einer EU, die durch eine prozesshafte Integrationsentwicklung gekennzeichnet ist und deren Parlament ebenso prozesshaft einen kontinuierlichen Stellungs- und Legitimationszuwachs durchläuft, der sich auch - aber nicht nur - im geschriebenen Recht niederschlägt. ${ }^{51}$ So ist die gesamte diesbezügliche Argumentation des Senats von einem geradezu bemühten „Kleinreden“ der Bedeutung des EP geprägt: Obwohl das Parlament den Kommissionsprä-

47 Vgl. Udo Di Fabio / Rudolf Mellinghoff, a.a.O. (Fn. 26), Rn. 158; Rainer Wernsmann, a.a.O. (Fn. 6), S. 26, S. 28; Hergen Eilert, Anmerkung zum Urteil des BVerfG vom 9.11.2011 - 2 BvC 4/10, 6/10, 8/10, in: DVBl. 2012, S. 234 - 235, S. 235; Martin Morlok, a.a.O. (Fn. 29), S. 79; Sebastian Roßner, a.a.O. (Fn. 4), S. 24.

48 Walter Frenz, Die Verfassungskonformität der 3-Prozent-Klausel für Europawahlen, in: NVwZ 2013, S. 1059 - 1062, S. 1062; Christoph Schönberger, a.a.O. (Fn. 15), S. 82 f., spricht von einer „Einebnung der grundgesetzlichen Maßstäbe“.

49 Sebastian Roßner, a.a.O. (Fn. 4), S. 24.

50 Vgl. Peter Müller, a.a.O. (Fn. 27), Rn. 26.

51 Vgl. RudolfWendt, a.a.O. (Fn. 8), S. 447; im Gegensatz zu heute hat das Gericht 1979 - bei noch wesentlich geringeren Kompetenzen des EP - dessen große faktische Bedeutung für die Entwicklung der damaligen EG ausdrücklich anerkannt, vgl. BVerfGE, Bd. 51, S. 222, S. 242: „Bleibt dem Europäischen Parlament nach alledem im Rahmen seiner beratenden Tätigkeit auch eine echte Mitentscheidung versagt, so kommt dieser doch für das Funktionieren und die Fortentwicklung der Europäischen Gemeinschaften eine große praktische Bedeutung zu; sie erweist sich etwa daran, dass die vom Europäischen Parlament beschlossenen Änderungsvorschläge in den meisten Fällen von der Kommission übernommen werden." 
sidenten wählt - was bei der Wahl 2014 mit der Nominierung von Spitzenkandidaten durch die großen Parteifamilien einen weiteren Politisierungs- und Legitimationsschub ausgelöst hat -, obwohl es die Kommission als Gesamtgremium billigen muss und (ungeachtet des noch sehr hohen Mehrheitserfordernisses) über das Instrument eines Misstrauensvotums (Art. 234 AEUV) verfügt, werden Regierungsbildungs- und -tragungsfunktion rundweg verneint. Ebenso wird beim Gesetzgebungsverfahren rein formal danach gesucht, ob es irgendeine Fallkonstellation gibt, bei der auch ohne das Parlament ein Rechtsakt in Kraft gesetzt werden kann - unter Missachtung des vertraglich gewollten Regelfalls einer aktiven parlamentarischen Mitwirkung und unabhängig von der vom Gericht selbst angemahnten „politischen Wirklichkeit“. Dabei verläuft der politische Prozess doch umgekehrt: Das Parlament ist durch den Vertrag von Lissabon im Gesetzgebungsprozess weiter gestärkt worden, was zwangsläufig angesichts der europäischen und demokratischen Dynamik dieses Prozesses dazu führt, dass Rechtsakte - und gar Haushalte - ohne parlamentarische $\mathrm{Zu}$ stimmung an Legitimation und demokratischer Akzeptanz einbüßen. Gerade eine Orientierung an der politischen Wirklichkeit würde es erforderlich machen, weniger in SchwarzWeiß-Kategorien denn in Grauschattierungen zu denken. Dann aber würde deutlich werden, dass die Grautöne zunehmend heller werden und dieser Prozess - nicht zuletzt wegen Art. 23 Abs. 1 GG - auch verfassungsgerichtliche Unterstützung verdient. ${ }^{52}$ Demgegenüber setzt der Senat seine im Lissabon-Urteil angelegte Rechtsprechung, das EP nicht als Parlament, sondern nur als „eine Vertretung der Völker der Mitgliedstaaten“ anzusehen $^{53}$, in einer verschärften Weise fort, die einer gegen die EU und ihre Institutionen gerichteten Grundstimmung entspricht. ${ }^{54}$ Damit aber befeuert das BVerfG Spekulationen, der wahre Grund für diese Judikatur liege in der Sorge eines eigenen Bedeutungsverlusts namentlich durch Einbindung in einen Gerichtsverbund unter Führung des EuGH - in einer enger zusammengewachsenen und stärkeren EU. 55

52 Vgl. Udo Di Fabio / Rudolf Mellinghoff, a.a.O. (Fn. 26), Rn. 158; Christoph Schönberger, a.a.O. (Fn. 15), S. 85; Jörg Geerlings / Andreas Hamacher, a.a.O. (Fn. 26), S. 677 f.

53 BVerfGE, Bd. 123, S. 267, S. 373, Rn. 284; dazu kritisch Martin Nettesheim, Die Lissabon-Entscheidung des Bundesverfassungsgerichts - Grundgesetzliche Grenzen der Integration, in: Europäisches Zentrum für Föderalismus-Forschung Tübingen (Hrsg.), Jahrbuch des Föderalismus 2010, Baden-Baden 2010, S. 403 - 413, S. 407 f.

54 Dieser Gesichtspunkt ist nicht nur in der juristischen Fachwelt - zum Beispiel spricht Christoph Schönberger, a.a.O. (Fn. 15), S. 80 von einem „Beleg für eine gestörte Beziehung“ zwischen BVerfG und europäischer Integration - thematisiert worden. So sind die Reaktionen aus dem EP selbst auf das Drei-Prozent-Urteil äußerst harsch ausgefallen, vgl. die Äußerungen von Rebecca Harms (MdEP) und Elmar Brok (MdEP), „Karlsruhe: Dreiprozenthürde verfassungswidrig“, in: FAZ vom 27. Februar 2014, S. 1; bereits nach dem Fünf-Prozent-Urteil hatte der ehemalige EPPräsident Hans-Gert Pöttering, „Karlsruhe und die europäische Dynamik“, in: FAZ vom 19. November 2011, S. 9 in einem Leserbrief die „Reserviertheit gegenüber der EU“ im BVerfG beklagt. Aber auch über die Betroffenen hinaus gibt es starke öffentliche Kritik, vgl. etwa Ulrich Beck im FAZ-Interview, „Höchstrichterlicher Populismus“, in: FAZ vom 28. Februar 2014, S. 37.

55 Vgl. Vassilios Skouris, Es gibt nicht nur Karlsruhe, in: FAZ vom 22. September 2011, S. 8; Martin Nettesheim, Die Karlsruher Verkündigung - Das BVerfG in staatsrechtlicher Endzeitstimmung, in: Armin Hatje / Jörg Philipp Terhechte (Hrsg.), Grundgesetz und europäische Integration. Die Europäische Union nach dem Lissabon-Urteil des Bundesverfassungsgerichts, EuR-Beiheft 1/2010, Baden-Baden 2010, S. 101 - 122, S. 104 ff.; Georg Paul Hefty, Absichtliche Atomisierung, in: FAZ vom 11. November 2011, S. 1; Ulrich Beck, a.a.O (Fn. 54). 


\section{3. Übertragung der Rechtsprechung zur Fünf-Prozent-Sperrklausel auf die Drei-Prozent-Hürde}

\subsection{Argumentation des Gesetzgebers}

Nach der Entscheidung des Bundesverfassungsgerichts vom 9. November 2011, die FünfProzent-Hürde im Europawahlrecht zu verwerfen, hat der Gesetzgeber auf Initiative aller Bundestagsfraktionen mit Ausnahme der Linken in einem neugefassten $\$ 2$ Abs. 7 EuWG eine auf drei Prozent abgesenkte Sperrklausel für die Wahl zum EP festgesetzt. Zentraler Anknüpfungspunkt für dieses als „Trotzreaktion“"56 oder „Machtkampf"57 bezeichnete legislative Manöver war die Aussage des BVerfG, dass die Zulässigkeit von Sperrklauseln zu keiner Zeit „ein für allemal“ festzustellen sei, sondern wesentliche Veränderungen tatsächlicher oder rechtlicher Art eine andere Beurteilung nötig machen könnten. ${ }^{58}$ Daher haben die Initianten in der Begründung des Gesetzentwurfs zwei Argumentationslinien aufgebaut.

So hat das EP - erstens - in einer Entschließung vom 22. November 2012 die Mitgliedstaaten aufgefordert, von der Ermächtigung des Art. 3 DWA Gebrauch zu machen und „in ihrem Europawahlrecht geeignete und angemessene Mindestschwellen für die Zuteilung der Sitze festzulegen, um dem in den Wahlen zum Ausdruck gekommenen Wählerwillen gebührend Rechnung zu tragen, bei gleichzeitiger Wahrung der Funktionalität des Parlaments "59. In den vorangestellten Erwägungen betont das EP seine Stellung als unmittelbares Vertretungsorgan der Unionsbürger, als Wahlorgan für den Kommissionspräsidenten und als Bestätigungsorgan der Gesamtkommission sowie die Verantwortlichkeit der Kommission gegenüber dem EP. Es ist wohl wenig spekulativ, darin auch eine Antwort des EP auf das Karlsruher Urteil zur Fünf-Prozent-Hürde zu sehen. Diese Aufforderung an die nationalen Wahlrechtsgesetzgeber lag 2011 noch nicht vor und sollte mit dem neugefassten $\$ 2$ Abs. 7 EuWG umgesetzt werden.

Zugleich hat das EP - zweitens - in dieser Entschließung die „europäischen politischen Parteien nachdrücklich auf[gefordert], Kandidaten für das Amt des Präsidenten zu nominieren“, die dann „im parlamentarischen Wahlkampf eine führende Rolle spielen“, indem sie den Wahlkampf ihrer Parteienfamilien anführen. Das EP „hält es für äußerst wichtig, die politische Legitimität sowohl des Parlaments als auch der Kommission zu stärken, indem deren Wahl jeweils unmittelbarer mit der Entscheidung der Wähler verknüpft wird“, weshalb es weiter gefordert hat, „dass möglichst viele Mitglieder der nächsten Kommission aus den Reihen des Europäischen Parlaments gestellt werden, um das Gleichgewicht zwischen den beiden Kammern der Legislative widerzuspiegeln“. Aus diesen Forderungen hat der deutsche Gesetzgeber abgeleitet, dass „sich eine verfassungsrechtlich erhebliche Entwicklung ab[zeichnet], die auf eine stärkere ,antagonistische Entwicklung von Regierung und Opposition“ hinausläuft" und so 2011 noch nicht konkret absehbar war. ${ }^{60}$ Die stärkere

56 Günter Nonnenmacher, a.a.O. (Fn. 24).

$57 \mathrm{Vgl}$. Helene Bubrowski, Das Wohl der Kleinen gegen das Wohl des Europäischen Parlaments, in: FAZ vom 19. Dezember 2013, S. 3.

58 Vgl. BVerfGE, Bd. 129, S. 300, S. 322, Rn. 90; BT-Drs. 17/13705, S. 6.

59 EP-Entschließung 2012/2829 (RSP), http://www.europarl.europa.eu/sides/getDoc.do?pub Ref=//EP//TEXT+TA+P7-TA-2012-0462+0+DOC+XML+V0//DE (Abruf am 29. März 2014); ebenso in BVerfG, a.a.O. (Fn. 2), Rn. 10.

60 BT-Drs. 17/13705, S. 6. 
Personalisierung der EP-Wahl durch europäische Spitzenkandidaten der Parteien verstärkt ihren Charakter als politische Richtungsentscheidung im Sinne einer Polarisierung zwischen den beiden großen Parteienfamilien, was zum einen die politische Bedeutung des EP im politischen Prozess weiter erhöht und zum anderen eine Veränderung der Mehrheitsbildungsprozesse im EP zur Folge hat. Während nämlich bisher die Mehrheit häufig durch ein Zusammenwirken der beiden großen Fraktionen erreicht wurde, wird nun jede der beiden großen Fraktionen bei den übrigen Fraktionen eine eigene Mehrheit zu erreichen versuchen. Eine deutlich höhere Zahl an fraktionslosen Abgeordneten - wie sie beim Fehlen einer Sperrklausel zu erwarten sind - wird diese politisierte Form der Mehrheitssuche bis hin zur Gefahr einer Blockade erheblich erschweren. ${ }^{61}$

Im verfassungsgerichtlichen Verfahren hat der Bundestag neben diesen Gesichtspunkten betont, dass der Gesetzgeber mit der Höhe von drei Prozent eine auf die Bedürfnisse des EP zugeschnittene Sonderlösung geschaffen hat, die erheblich leichter zu überwinden ist und deshalb eine erheblich verringerte Eingriffsintensität in die Wahlrechtsgleichheit aufweist. Insbesondere können auch Parteien, die nicht im Bundestag vertreten und daher an der Festlegung der Sperrklausel nicht beteiligt sind, diese Hürde überspringen. ${ }^{62}$

\subsection{Reaktion des BVerfG}

In konsequenter Fortführung seiner Sperrklausel-Rechtsprechung seit 2008 stellt das Gericht zunächst fest, dass auch bei einer Drei-Prozent-Hürde eine Ungleichbehandlung von Wählerstimmen vorliegt. ${ }^{63}$ Auch hier gibt es Stimmen (für Parteien unter drei Prozent), die gerade wegen der Existenz der Sperrklausel ohne Erfolgswert bleiben, während sich andere Stimmen (für Parteien ab drei Prozent) in der Zusammensetzung des Parlaments (genauer: des deutschen Abgeordnetenkontingents) niederschlagen. Aus Karlsruher Sicht ist auch der mit einer Drei-Prozent-Hürde verbundene Eingriff in die Wahlrechtsgleichheit nicht zu rechtfertigen.

Zum einen handelte es sich bei der EP-Entschließung vom 22. November 2012 lediglich um einen „rechtlich unverbindlichen Appell an die Mitgliedstaaten“, durch den sich weder tatsächlich noch rechtlich etwas geändert hat. Insbesondere sei nach wie vor nicht erkennbar, dass auf europarechtlicher Ebene - etwa durch Änderung des DWA - eine Sperrklausel verbindlich vorgegeben werden soll. ${ }^{64}$

Zum anderen steckte die vom Gesetzgeber betonte Entwicklung zu einer antagonistischen Profilierung von Regierung und Opposition auf europäischer Ebene „noch in den Anfängen “; ob sich daraus tatsächlich veränderte Funktionsbedingungen des EP in der nächsten Wahlperiode ergeben, sei noch nicht hinreichend konkret abzusehen. ${ }^{65}$ Vielmehr sei die Stärkung der politischen Legitimität von EP und Kommission schon deshalb nicht ersichtlich, weil keine primärrechtlichen Kompetenzverschiebungen in diesem Sinne angestrebt würden. ${ }^{66}$ Deshalb erscheinen die mit der Politisierung des Kommissionspräsidenten

61 Vgl. ebenda.

62 Vgl. BVerfG, a.a.O. (Fn. 2), Rn. 21, Rn. 23, Rn. 26.

63 Vgl. BVerfG, a.a.O. (Fn. 2), Rn. 46 f., Rn. 51.

64 BVerfG, a.a.O. (Fn. 2), Rn. 67; vgl. auch Rainer Wernsmann, a.a.O. (Fn. 6), S. 27.

65 BVerfG, a.a.O. (Fn. 2), Rn. 70, Rn. 72.

66 Vgl. BVerfG, a.a.O. (Fn. 2), Rn. 73. 
verbundenen Erwartungen einer politischen Stärkung des EP und der Entwicklung zu einem Regierungs-Oppositions-Dualismus dem BVerfG „spekulativ“ und „ungewiss“.67 Auch die „Zahl von künftig möglicherweise achtzig kooperationsunwilligen Abgeordneten lässt sich angesichts derartiger Unsicherheiten nicht mit der notwendigen Wahrscheinlichkeit prognostizieren“, weil viele Einzelabgeordnete in den vorhandenen oder einer neu gegründeten Fraktion Aufnahme finden können, selbst wenn „die Bereitschaft einer Fraktion, Abgeordnete aufzunehmen, die auf nationaler Ebene als Konkurrenten auftreten, abnehmen könnte“68. Hinzu kommt, dass gerade kleine Parteien zur Veränderungsoffenheit des politischen Prozesses einen wertvollen Beitrag leisten können. ${ }^{69}$ Sollte sich aber wirklich erweisen, dass das Fehlen einer Sperrklausel die Funktionsfähigkeit des EP beeinträchtigt, besteht zudem jederzeit die Möglichkeit der Korrektur, da nicht das dann eventuell handlungsunfähige EP selbst, sondern der davon unberührte deutsche Wahlrechtsgesetzgeber dafür zuständig wäre; eine Art Präventiv-Sperrklausel stellt jedenfalls aus Sicht des BVerfG eine unverhältnismäßige Vorverlagerung des Eingriffs in die Wahlrechtsgleichheit dar. ${ }^{70}$

Mit der deutlichen Sperrklausel-Absenkung von fünf auf drei Prozent setzt sich das Gericht nicht näher auseinander, denn der Senat verneint bei dieser Sperrklausel - wie bei der Fünf-Prozent-Hürde zuvor - das Vorliegen eines Rechtfertigungsgrundes für den Eingriff in die Wahlrechtsgleichheit „bereits dem Grunde nach“. Damit ist der Eingriff unabhängig davon, ob er mit höherer (fünf Prozent) oder geringerer (drei Prozent) Intensität erfolgt, unzulässig. ${ }^{71}$

\subsection{Kritik: Weitere Verschärfung der Rechtfertigungshürde und Idealisierung der Splitterparteien}

Das gesetzgeberische Bemühen, nicht einmal zwei Jahre nach der sehr grundsätzlich angelegten Entscheidung des BVerfG zur Fünf-Prozent-Hürde eine lediglich in ihrer Höhe modifizierte $^{72}$ Sperrklausel einzuführen und gleichzeitig wesentliche Veränderungen gegenüber 2011 darzutun, ist wenig überzeugend. ${ }^{73}$ Grund dafür ist der innere Widerspruch im Gebaren des Gesetzgebers, sich zum einen der Diktion des BVerfG zu unterwerfen, zum ande-

67 BVerfG, a.a.O. (Fn. 2), Rn. 75, Rn. 78.

68 BVerfG, a.a.O. (Fn. 2), Rn. $80-82$.

69 Vgl. BVerfG, a.a.O. (Fn. 2), Rn. 76.

70 Vgl. BVerfG, a.a.O. (Fn. 2), Rn. 63.

71 BVerfG, a.a.O. (Fn. 2), Rn. 83; bereits vor der Entscheidung ebenso Rainer Wernsmann, a.a.O. (Fn. 6), S. 26 f.

72 Denkbar wäre auch die Verknüpfung einer Sperrklausel mit einer Alternativstimme gewesen, was das BVerfG zumindest zu einer differenzierten Bewertung der Frage des Vorliegens eines Eingriffs in die Wahlrechtsgleichheit (allerdings nicht in die Chancengleichheit der Parteien) genötigt hätte; vgl. zu dieser Thematik Matthias Damm, Die Nebenstimme bei Bundestagswahlen: Wer A sagt, darf auch B sagen?, in: DÖV 2013, S. 913 - 920; kritisch dazu Rudolf Wendt, a.a.O. (Fn. 8), S. $455 \mathrm{f}$.

73 Vgl. Walter Frenz, a.a.O. (Fn. 48), S. 1060 f., nennt - anders als der Gesetzgeber - noch die zwischen 2011 und 2013 „grundlegend verschärfte Eurokrise“ als wesentliche tatsächliche Veränderung, relativiert allerdings das Argument zu Recht mit dem Hinweis, dass das EP bei der Krisenbewältigung nur eine Zuschauerrolle eingenommen hat, weil dafür der Rat zuständig ist (Art. 136 AEUV). 
ren aber das Urteil zur Fünf-Prozent-Hürde politisch nicht wirklich akzeptieren zu wollen. Hinzu kommt, dass die in der Gesetzesbegründung herangezogene Aussage des BVerfG von 2011 zur zeitlichen Relativität von Zulässigkeitsentscheidungen über Sperrklauseln - und damit der Abänderungsmöglichkeit bei wesentlichen Veränderungen - zunächst (nur) den gegenteiligen Fall betraf, also die dauerhafte Überprüfungspflicht, ob Rechtfertigungsgründe für den Eingriff in die Wahlrechtsgleichheit bei einer zulässigerweise bestehenden Sperrklausel fortbestehen. Folglich überrascht es nicht, dass der in der Sperrklauselthematik mehrheitlich sehr festgelegte Senat weder eine rechtlich unverbindliche Appell-Resolution des (betroffenen) EP noch die weitere Politisierung und Polarisierung der bislang vor allem administrativ wahrgenommenen europäischen Ebene zum Anlass einer Korrektur seiner Rechtsprechung genommen hat.

Das bedeutet jedoch nicht, dass das jüngste Sperrklausel-Urteil aus Karlsruhe keinen weiteren Anlass zur Kritik bieten würde. So werden die im Urteil zur Fünf-Prozent-Hürde schon bis zur Funktionsunfähigkeit verengten Anforderungen an die Funktionsbeeinträchtigung dadurch noch verschärft, dass der für notwendig gehaltene Grad der Wahrscheinlichkeit ihres Eintritts weiter erhöht wird. Es reicht nun nicht mehr eine (äußerst nachvollziehbare $^{74}$ ) Prognose, sondern die Funktionsstörung muss so konkret absehbar sein, dass sie mehr oder weniger unmittelbar bevorsteht. So hat das Gericht die vom Gesetzgeber nach politisch-realer Lebenserfahrung erwarteten Entwicklungen im Institutionengefüge und Funktionsstörungen im EP schon deshalb als nicht hinreichend absehbar eingestuft, weil - nach eher theoretischen Erwägungen des Senats (namentlich zur Integrationsfähigkeit und -bereitschaft der Fraktionen) - auch andere Zukunftsszenarien denkbar sind. Damit aber wird die gesetzgeberische Einschätzungsprognose durch eine vom Gericht geforderte (polizeirechtlich anmutende) Gefahrenlage ersetzt. ${ }^{75}$ Dies bewirkt zugleich eine zeitliche Verlagerung der Rechtfertigungsmöglichkeit einer Sperrklausel nach hinten, was die Gefahr weiter erhöht, dass das Kind frühestens nach seinem Fall in den Brunnen - sprich: eingetretenen Funktionsstörungen wie einer Entscheidungsblockade - zu retten sein wird. Da hilft es auch nichts, dass - wie das BVerfG betont - nicht das EP selbst, sondern der deutsche Gesetzgeber durch die Wiedereinführung einer Sperrklausel mit der Rettungsmaßnahme betraut wird. Denn dann sind die hürdenlose Wahl und die Mandatszuteilung bereits erfolgt, und eine Änderung des EuWG könnte erst zur nächsten EP-Wahl ihre korrigierende Wirkung entfalten. Für die Dauer der laufenden Wahlperiode hingegen müsste das EP mit den Funktionsstörungen nolens volens leben, was weder Art. 3 und 21 GG gebieten, noch Art. 23 Abs. 1 GG zulässt. ${ }^{76}$

Außerdem ist die sehr konstruktive - ja beinahe idealisierende - Rolle zu hinterfragen, die das BVerfG den Splitterparteien für den politischen Prozess beimisst. So ist die Integrationsleistung größerer Parteien schon deshalb sehr viel höher, weil zahlreiche divergierende Anliegen der Bevölkerung wegen der hohen Meinungspluralität in größeren Parteien bereits innerparteilich zum Ausgleich gebracht werden müssen. ${ }^{77}$ Daraus folgt, dass Parteien,

74 Vgl. Peter Müller, a.a.O. (Fn. 27), Rn. $14 \mathrm{ff}$.

75 Das Gericht wendet sich demgemäß auch gegen die „Zubilligung von weitgehend frei ausfüllbaren Prognosespielräumen“ für den Gesetzgeber, weil „es bei der Wahlgesetzgebung um die Grundbedingungen politischer Konkurrenz geht“, vgl. BVerfG, a.a.O. (Fn. 2), Rn. 64.

76 Vgl. Peter Müller, a.a.O. (Fn. 27), Rn. 11.

77 Vgl. Rudolf Wendt, a.a.O. (Fn. 8), S. 451. 
die einen nennenswerten Teil der Bevölkerung ansprechen wollen, „durch die Pluralität der Anliegen der Wählerschaft zur Orientierung am Gemeinwohl genötigt" werden. ${ }^{78}$ Demgegenüber sind kleine Parteien mit geringer Bevölkerungsresonanz häufig auf einseitige und partikulare Interessen fokussiert ${ }^{79}$ und mit Rücksicht auf die eigene Klientel zu einem übergeordneten Interessenausgleich gerade nicht bereit; damit erfüllen diese Parteien die Verfassungserwartung einer gemeinwohlorientierten Mandatsausübung (vgl. Art. 38 Abs. 1 S. 2 GG) tendenziell weniger. ${ }^{80}$ Dann aber überzeugt der Karlsruher Ansatz nicht, dass das GG diese Form der Mandatsausübung unter Zurückstellung der Funktionsfähigkeit des Parlaments besonders geschützt sehen möchte.

\section{Problematisches Rollenverständnis des BVerfG sowohl auf der Zeitachse als auch im Staatsaufbau}

\subsection{Fehlende Geradlinigkeit und Folgerichtigkeit gegenüber älterer Judikatur}

Gerade auf dem politisch so sensiblen Feld des Wahlrechts bricht der Zweite Senat des BVerfG in der jüngeren Vergangenheit mit der früheren Rechtsprechung des Gerichts. ${ }^{81}$ Dies gilt für die Überhangmandate, die zwar stets problematisiert, aber zuletzt 1997 noch im Umfang von circa fünf Prozent für zulässig gehalten und in einer Entscheidung von 2008 trotz Relevanz durch Schweigen (einstimmig) gebilligt wurden, bevor der Senat 2012 die unausgeglichenen Überhangmandate (wiederum einstimmig) für hochgradig problematisch und allenfalls in der Höhe von 2,5 Prozent für vertretbar erklärt hat. ${ }^{82}$ Ebenso hat der Senat das Problem des negativen Stimmgewichts in einer früheren Entscheidung - ebenfalls trotz Betroffenheit - nicht angesprochen und die Wahlprüfungsbeschwerden als „offensichtlich unbegründet" verworfen, um dann 2008 darin einen eklatanten Verfassungsverstoß zu erkennen. ${ }^{83}$ Und schließlich hat der Senat die gesetzgeberische Behandlung der Auslandsdeutschen in ihrer Zielsetzung des Öfteren gebilligt, bevor er 2012 plötzlich die

78 Vgl. ebenda, S. 452.

79 Unter den sieben 2009 an der Fünf-Prozent-Hürde gescheiterten Parteien befinden sich die Tierschutzpartei, die Familien- und die Rentner-Partei, vgl. http://www.bundeswahlleiter.de/de/europawahlen/EU_BUND_09/ergebnisse/bundesergebnisse/ (Abruf am 29. März 2014); vgl. auch Johannes Leithäuser, Der Jubel der Zersplittergruppen, in: FAZ vom 27. Februar 2014, S. 3.

80 Vgl. RudolfWendt, a.a.O. (Fn. 8), S. 452; vgl. auch BVerfGE, Bd. 51, S. 222, S. 236.

81 Vgl. die instruktive Zusammenstellung von Gerd Strohmeier, Kann man sich auf Karlsruhe verlassen? Eine kritische Bestandsaufnahme am Beispiel des Wahlrecht, in: ZParl, 44. Jg. (2013), H. 3, S. 629-644, auf die bezüglich der Einzelheiten zur Vermeidung von Wiederholungen verwiesen wird; auch Jörn Ipsen, Wahlrecht im Spannungsfeld von Politik und Verfassungsgerichtsbarkeit, in: DVBl. 2013, S. 265 - 274, S. 273, beklagt trotz inhaltlicher Zustimmung zur restriktiven neuen Linie die "Schwankungen“ in der Wahlrechtsjudikatur des BVerfG.

82 Vgl. BVerfGE, Bd. 95, S. 335 (10. April 1997); BVerfGE, Bd. 121, S. 266 (3. Juli 2008); BVerfGE, Bd. 131, S. 316 (25. Juli 2012); vgl. Gerd Strohmeier, a.a.O. (Fn. 81), S. 630 ff.; Jörn Ipsen, a.a.O. (Fn. 81), S. 268; Volker M. Hang, Das Bundesverfassungsgericht als Gesetzgeber anstelle des Gesetzgebers: Ein kritischer Blick auf das Wahlrechtsurteil vom 25. Juli 2012, in: ZParl., 43. Jg. (2012), H. 3, S. 658 - 674, S. 667 ff.

83 Vgl. BVerfGE, Bd. 121, S. 266 (3. Juli 2008); vgl. Gerd Strohmeier, a.a.O. (Fn. 81), S. 634 ff. mit weiteren Nennungen. 
alleinige Anknüpfung an einen früheren mindestens dreimonatigen Aufenthalt in Deutschland als einen Verstoß gegen den Grundsatz der allgemeinen Wahl eingestuft hat. ${ }^{84}$ Dieser Anhäufung von „beinahe artistischen Kehrtwenden“85 im Wahlrecht ist auch die Sperrklausel-Rechtsprechung hinzuzufügen.

So hat das BVerfG in einer einstimmigen Entscheidung 1979 die Verfassungsmäßigkeit der Fünf-Prozent-Klausel im Europawahlrecht ausdrücklich bejaht. In diesem den Handlungsspielraum des Gesetzgebers ausdrücklich respektierenden Beschluss ${ }^{86}$ betont das Gericht sowohl die Aufgabe des Wahlrechts, „ein funktionsfähiges Organ hervorzubringen“, als auch die Bedeutung und den erheblichen faktischen Einfluss des EP als Vertretung der Völker mit einer wichtigen Integrationsfunktion, obwohl damals noch der Rat „das hauptsächliche Entscheidungs- und Gesetzgebungsorgan“ war und die Bildung der Kommission „ohne Beteiligung des Parlaments durch die Regierungen der Mitgliedstaaten“ erfolgt ist. ${ }^{87}$ Für eine verfassungsrechtliche Beanstandung der Fünf-Prozent-Hürde „müssten besondere Umstände des Einzelfalles vorliegen, die ein solches Quorum unzulässig machen würden" 88 . Gegenüber dieser Entscheidung stellt sich die Sperrklausel-Rechtsprechung des heutigen Zweiten Senats sowohl im Ergebnis als auch in der Begründung diametral entgegengesetzt dar. Zwar gibt es keine Bindung des BVerfG an seine frühere Rechtsprechung, weil mit veränderten Verhältnissen natürlich auch Modifikationen in der Judikatur möglich sein müssen. Um aber als folgerichtig überzeugen zu können, muss ein Wandel in der Rechtsprechung mit wesentlichen Veränderungen tatsächlicher oder rechtlicher Art korrespondieren. ${ }^{89}$ Es müssten also Umstände eingetreten sein, die Sperrklauseln heute wesentlich entbehrlicher erscheinen ließen, als das 1979 der Fall war. Vergleicht man die Sachund Rechtslage des EP im Jahr 1979 mit der des Jahres 2011 beziehungsweise 2014, zeigt sich jedoch das genaue Gegenteil:

- Während 1979 insgesamt 410 Abgeordnete aus neun Mitgliedstaaten das EP bildeten ${ }^{90}$, sind es nun 751 Parlamentarier aus 28 Staaten. ${ }^{91}$ Es ist offensichtlich, dass diese qualitative und quantitative Vergrößerung des Parlaments dessen interne Komplexität erheblich gesteigert hat - mit unmittelbaren und erheblichen Folgen sowohl für die Integrationsfähigkeit der Fraktionen als auch für die Willens- und Mehrheitsbildung.

- Außerdem hat sich das EP seit 1979 von einem reinen Beratungsgremium ohne eigene legislative Funktionen ${ }^{92}$ zu einem wichtigen Mitentscheidungsorgan der EU entwickelt, indem es etwa an der Legitimation der Kommission wesentlichen Anteil hat und gemeinsam mit dem Rat über die Gesetzgebung entscheidet (Art. 14 EUV). ${ }^{93}$ Durch

84 Vgl. BVerfGE, Bd. 132, S. 39; Gertrude Lübbe-Wolf, Abweichende Meinung, in: BVerfGE, Bd. 132, S. 39, S. 60 ff., betont einleitend das überraschende Entscheidungsergebnis und den damit verbundenen Orientierungsverlust; vgl. auch Gerd Strohmeier, a.a.O. (Fn. 81), S. 639 ff.

85 Gerd Strohmeier, a.a.O. (Fn. 81), S. 643.

86 Vgl. BVerfGE, Bd. 51, S. 237 f.

87 Ebenda, S. 222, S. 236, S. 239, S. 241, S. 245.

88 Ebenda, S. 222, S. 237.

89 Vgl. auch BVerfGE, Bd. 129, S. 300, S. 321 f., Rn. 90.

90 Vgl. BVerfGE, Bd. 51, S. 222, S. 223.

91 Normgröße gemäß Art. 14 Abs. 2 UAbs. 1 S. 2 EUV; zum Zeitpunkt des Urteils zur Drei-ProzentHürde waren es sogar (übergangsweise) 766 Abgeordnete, vgl. BVerfG, a.a.O. (Fn. 2), Rn. 7.

92 Vgl. BVerfGE, Bd. 51, S. 222, S. $241 \mathrm{f}$.

93 Was auch das Gericht ausdrücklich anerkennt, BVerfG, a.a.O. (Fn. 2), Rn. 4. 
diesen tiefgreifenden Aufgaben- und Kompetenzzuwachs ist die Bedeutung des EP und damit auch dessen Funktionsfähigkeit für die Handlungsfähigkeit des Gesamtverbundes EU - erheblich angestiegen.

- Des Weiteren besteht seit 2002 die europarechtliche Möglichkeit einer SperrklauselRegelung in Art. 3 DWA, woraus sich - wie ausgeführt - eine europarechtliche Zulässigkeit nationaler Sperrklauselregelungen im Zusammenspiel mit dem europarechtlichen Gleichheitssatz ergibt.

- Parallel zu diesen Entwicklungen hat in Deutschland die Wählerbeweglichkeit zugenommen, weshalb neue Parteien heute eine Sperrklausel leichter überwinden können als früher. ${ }^{94}$ Die Gefahr einer Verkrustung des Parteiensystems und damit eines „Wagenburg“-Charakters der Sperrklauselgesetzgebung durch die etablierten Parteien ist damit noch geringer, als sie ohnehin schon war. Dadurch reduziert sich die Eingriffsintensität in die Chancengleichheit der Parteien.

Keine dieser Entwicklungen ist auch nur im Ansatz geeignet, die heute wesentlich restriktivere Rechtsprechung gegenüber der EP-Sperrklausel zu rechtfertigen. ${ }^{95}$ Umgekehrt würde ein Schuh daraus: Hätte das BVerfG nicht bereits 1979 die Zulässigkeit der Sperrklausel bejaht, würden diese Gründe einen Rechtsprechungswandel zugunsten einer Sperrklausel ohne weiteres tragen können. Insofern handelt es sich bei den EP-Sperrklausel-Entscheidungen von 2011 und 2013 um einen Bruch in der Rechtsprechungskontinuität, wie er auch in den oben genannten anderen Bereichen des Wahlrechts zu konstatieren ist. Mit seiner Abwendung von der eigenen Vorgänger-Judikatur - ohne sich damit auch nur argumentativ auseinanderzusetzen ${ }^{96}$ - gibt der Senat ein weiteres Mal ein zentrales Gut der Rechtsprechung auf, denn in der Berechenbarkeit und Verlässlichkeit gerichtlicher Entscheidungen liegt der zentrale Beitrag der Justiz zum Rechtsfrieden.

\subsection{Kompetenzüberschreitung zum Nachteil des Gesetzgebers}

\section{(a) Argumentation des BVerfG}

Ausgehend von der unstreitig hohen Bedeutung des Wahlrechts für das Gelingen der demokratischen Staatsform, weil diese von der Akzeptanz der Mehrheit durch die Minderheit lebt ${ }^{97}$, reklamiert das BVerfG für sich auf diesem Feld eine „strikte verfassungsgerichtliche Kontrolle“. Zur Begründung wird geltend gemacht, dass „mit Regelungen, die die Bedingungen der politischen Konkurrenz berühren, die parlamentarische Mehrheit gewissermaßen in eigener Sache tätig wird und gerade bei der Wahlgesetzgebung die Gefahr besteht,

94 Udo Di Fabio / Rudolf Mellinghoff, a.a.O. (Fn. 26), Rn. 155; dies zeigt auch das Abschneiden der AfD bei der Europawahl 2014, die auch bei Bestehen einer Sperrklausel in das EP eingezogen wäre.

95 Siehe auch Rainer Wernsmann, a.a.O. (Fn. 6), S. 25; Christoph Schönberger, a.a.O. (Fn. 15), S. 85; Hergen Eilert, a.a.O. (Fn. 47), S. 235.

96 Dazu kritisch Udo Di Fabio / Rudolf Mellinghoff, a.a.O. (Fn. 26), Rn. 148; Christoph Schönberger, a.a.O. (Fn. 15), S. 85.

97 Für diese nicht zu überschätzende Bedeutung sind im Schrifttum anschauliche Metaphern gefunden worden: „Herzkammer der Demokratie“ von Henner Jörg Boehl, zitiert bei Gerd Strohmeier, a.a.O. (Fn. 81), S. 629; „Wechselstube der Demokratie“, in der politische Zustimmung in staatliche Macht eingetauscht wird, von Sebastian Roßner, a.a.O. (Fn. 4), S. 22. 
dass die jeweilige Parlamentsmehrheit sich statt von gemeinwohlbezogenen Erwägungen vom Ziel des eigenen Machterhalts leiten lässt"; dies soll allerdings nicht so weit gehen, dass das BVerfG anstelle des Gesetzgebers dessen Aufgabe übernimmt und dessen Zweckmäßigkeitsbeurteilungen durch eigene ersetzt. ${ }^{98}$ Dieses Rollenverständnis als „Hüter des politischen Wettbewerbs“ hat auch in der Literatur teilweise Zustimmung gefunden. ${ }^{99}$

\section{(b) Kritik: Überschreitung der Kompetenzgrenzen}

Natürlich wäre es naiv zu glauben, dass politische Akteure bei der verbindlichen Festsetzung politischer Spielregeln - insbesondere zur Verteilung von Macht - völlig neutral zu Werke gingen und dabei in keiner Weise mittelbare oder unmittelbare Eigeninteressen verfolgten. ${ }^{100}$ Doch überhöht der Senat diesen Umstand zu einem Generalverdacht, der so klingt, als ob objektive Belange - wie etwa die Funktionsfähigkeit des Parlaments - allenfalls vorgeschützt würden und von der Politik nicht als Eigenwert angesehen würden. Eine solche Bewertung mag angesichts der vorherrschenden Parteien- und Politikverdrossenheit sowie des äußerst geringen sozialen Ansehens von Politikern einer weit verbreiteten Stimmung in der Bevölkerung entgegenkommen, wird aber in seiner Pauschalität dem Engagement und der Motivation vieler haupt- und ehrenamtlich in der Politik Tätigen nicht gerecht. ${ }^{101}$ Sie entspricht auch nicht dem Leitbild des Grundgesetzes vom gemeinwohlorientierten Mandatsträger gemäß Art. 38 Abs. 1 S. 2 GG. Zudem ist der Begründungstopos der „Entscheidung in eigener Sache“ im Sperrklauselrecht - anders als etwa bei Fragen der Abgeordnetenentschädigung - schief, da es zur Sperrklausel-Thematik keine kartellartige Homogenität in den Interessen der im Bundestag vertretenen Parteien gibt. ${ }^{102}$ Hinzu kommt, dass auch das BVerfG ,in eigener Sache“ entscheidet, ohne dabei eine besondere Sensibilität zu zeigen. So hat es die - im Schrifttum seit jeher stark kritisierte ${ }^{103}$ - bundestagsseitige Wahl der Bundesverfassungsrichter durch einen nur zwölfköpfigen Wahlausschuss (\$ 6 BVerfGG) mit Beschluss vom 19. Juni 2012 gebilligt: „Die Übertragung der Wahl der Bundesverfassungsrichter auf einen Wahlausschuss, dessen Mitglieder der Verschwiegenheitspflicht unterliegen ( $\$ 6$ Abs. 4 BVerfGG), findet ihre Rechtfertigung in dem erkennbaren gesetzgeberischen Ziel, das Ansehen des Gerichts und das Vertrauen in seine Unabhängigkeit zu festigen und damit seine Funktionsfähigkeit zu sichern. "104 Denn so bleibt den Richtern eine öffentliche und womöglich kritische Debatte ihrer Person meist erspart.

98 Vgl. BVerfGE, Bd. 129, S. 300, S. 322 f., Rn. 91; BVerfG, a.a.O. (Fn. 2), Rn. 59.

99 Vgl. Martin Morlok, a.a.O. (Fn. 29), S. 77; Jörn Ipsen, a.a.O. (Fn. 81), S. 265; Sebastian Roßner, a.a.O. (Fn. 4), S. $23 \mathrm{f}$.

100 Vgl. Martin Morlok, a.a.O. (Fn. 29), S. 79; RudolfWendt, a.a.O. (Fn. 8), S. 431.

101 Vgl. Hergen Eilert, a.a.O. (Fn. 47), S. 234.

102 Peter Müller, a.a.O. (Fn. 27), Rn. 10.

103 Vgl. Wolfgang Meyer, in: Ingo von Münch / Philip Kunig (Hrsg.), Grundgesetz Kommentar, München 2012, Art. 94, Rn. 9; der verfassungsrechtlichen Kritik ausdrücklich zustimmend der heutige Präsident des BVerfG Andreas Voßkuhle, in: Hermann von Mangoldt / Friedrich Klein / Christian Starck (Hrsg.), Kommentar zum Grundgesetz, München 2010, Band 3, Art. 94, Rn. 10.

104 BVerfGE, Bd. 131, S. 230, S. 236, Rn. 14; vgl. auch die rechtspolitische Argumentation bei Rupert Scholz, Pro \& Contra - Sollen Bundesverfassungsrichter vom Plenum gewählt werden?, in: ZRP 2012, S. 191. 
Doch selbst wenn eine solche pejorativ-einseitige Bewertung der Arbeit des Gesetzgebers verfassungsrechtlich angezeigt wäre, könnte dieser politisch-praktische Befund eine verfassungsgerichtliche Abänderung der grundgesetzlichen Kompetenzordnung nicht rechtfertigen. ${ }^{105}$ Danach ist es Aufgabe des Gesetzgebers, im Rahmen eines - je nach Größe der künftigen Unwägbarkeiten des Regelungsgegenstandes unterschiedlich weiten - Prognoseund Einschätzungsspielraums Gestaltungsentscheidungen zu treffen und die dafür erforderlichen Abwägungen vorzunehmen. Schließlich muss er dafür auch die Verantwortung gegenüber dem Volk als Souverän (spätestens bei der nächsten Wahl) übernehmen. Die verfassungsgerichtliche Kontrollkompetenz dagegen ist darauf beschränkt, offenkundig sachwidrige, völlig überzogene oder missbräuchliche Maßnahmen des Gesetzgebers zu beanstanden. ${ }^{106}$ Diese Aufgabenteilung korrespondiert auch mit der unterschiedlichen Qualität der demokratischen Legitimation. ${ }^{107}$ Hier aber tut das BVerfG genau das, was nach seinen eigenen Worten gerade nicht seine Aufgabe ist ${ }^{108}$ : Es ersetzt „die vertretbare Entscheidung des Gesetzgebers durch seine eigene vertretbare Entscheidung "109, etwa indem es Wahrscheinlichkeiten für bestimmte Entwicklungen dezidiert anders beurteilt als der Gesetzgeber. ${ }^{110}$ Mit dieser Verschiebung der Gewaltenteilungsgrenze zwischen Judikative und Legislative zu Lasten letzterer setzt Karlsruhe gerade im Wahlrecht eine zweifelhafte Tradition fort ${ }^{111}$ und entwickelt Deutschland wieder ein Stück weiter zu einem Richterstaat. ${ }^{112}$ Man darf füglich bezweifeln, dass das im Sinne des Grundgesetzes ist.

\section{Fazit und Ausblick: Sorge um die Sperrklauseln in Bund und Land}

Die Sperrklausel-Rechtsprechung des BVerfG zum Europawahlrecht begegnet vielfältigen rechtlichen Einwänden. Weder wird diese Judikatur der Aufgabe des Wahlrechts zur Schaffung einer funktions- und handlungsstarken Volksvertretung gerecht, noch entspricht sie - insbesondere durch die mehrfach auffällig national-exklusive Sichtweise - der Europafreundlichkeit des Grundgesetzes. Außerdem negiert sie geradezu bemüht die offensichtliche Bedeutung des EP als demokratisch unmittelbar legitimiertes Integrations- und Repräsentationsorgan von rund einer halben Milliarde Menschen bei einem gleichzeitig unpolitischen Verständnis der Arbeitsweise des EP und seiner Fraktionen. Schließlich nimmt der heutige Zweite Senat - keineswegs nur im Sperrklausel-Recht - erstaunlich

105 Vgl. Christoph Schönberger, a.a.O. (Fn. 15), S. 83.

106 Vgl. BVerfGE, Bd. 51, S. 222, S. 238; Udo Di Fabio / Rudolf Mellinghoff, a.a.O. (Fn. 26), S. 350 ff., Rn. 154 - 156; Peter Müller, a.a.O. (Fn. 27), Rn. 6; siehe auch Philipp Austermann, Die rechtlichen Grenzen des Bundesverfassungsgerichts im Verhältnis zum Gesetzgeber, in: DÖV 2011, S. $267 \mathrm{ff}$.

107 Zum Legitimationsaspekt bei nichtöffentlichen Richterwahlen im Richterwahlausschuss des Bundestags vgl. auch Christine Landfried, Pro \& Contra - Sollen Bundesverfassungsrichter vom Plenum gewählt werden, in: ZRP 2012, S. 191.

108 Vgl. Martin Morlok, a.a.O. (Fn. 29), S. 79; RudolfWendt, a.a.O. (Fn. 8), S. 431.

109 Peter Müller, a.a.O. (Fn. 27), Rn. 9.

110 Vgl. etwa BVerfG, a.a.O. (Fn. 2), Rn. $75-82$.

111 Vgl. Volker M. Haug, a.a.O. (Fn. 82), S. 673 f.

112 Vgl. zum Grundproblem, wenngleich in anderem Zusammenhang, Bernd Rüthers, Wer herrscht über das Grundgesetz, in: FAZ vom 18. November 2013, S. 7, und Martin Nettesheim, a.a.O. (Fn. 55), S. 411 f. (,gouvernement des juges“). 
wenig Rücksicht auf die Rechtsprechung seiner Vorgängerbesetzungen und auf die Zuständigkeitsabgrenzung zwischen Verfassungsgerichtsbarkeit und Gesetzgeber, dessen Interessen- und Motivationslage einseitig negativ dargestellt wird. Der Senat nimmt seine Interpretationshoheit bezüglich des Grundgesetzes hier in einem derart extensiven Maße wahr, dass es sich an die eigentliche Gesetzgebungstätigkeit stark annähert. Dadurch ergibt sich aus Sicht des Gesetzgebers eine Negativkombination von ungewöhnlich enger Steuerungsdichte und geringer Verlässlichkeit des Gerichts. So segensreich das Wirken des BVerfG insbesondere im Bereich der Grundrechte war und ist, so problematisch ist seine jüngere Rechtsprechung zum Wahlrecht und insbesondere zu den EP-Sperrklauseln.

Gleichzeitig setzt der Senat die Erosion von Sperrklauseln, die er in seiner Entscheidung zum schleswig-holsteinischen Kommunalwahlrecht begonnen hat, in einer derart strikten Weise fort, dass im politischen Raum bereits um den langfristigen Fortbestand der FünfProzent-Hürden bei Bundes- und Landtagswahl gefürchtet und sogar eine entsprechende Ergänzung des GG diskutiert wird. ${ }^{113}$ Zwar hat das BVerfG im Urteil zur Fünf-ProzentHürde noch eine klare Abgrenzung in der Rechtfertigung der Sperrklausel zugunsten des Bundestages vorgenommen ${ }^{114}$, doch ist insgesamt zu konstatieren, dass der Rechtsprechungstrend gegen die Sperrklauseln läuft, nachdem im Gegensatz zu früheren Urteilen desselben Gerichts zuerst die kommunale (2008) und dann die europäische Sperrklausel (2011/14) gefallen ist. Zudem fällt auf, dass die Rechtfertigung der Sperrklausel auf der nationalen Ebene im Urteil zur Drei-Prozent-Hürde nur noch in einer „kann“-Formulierung - mithin abgeschwächt - genannt wird. ${ }^{115}$ Berücksichtigt man schließlich die Wendigkeit des BVerfG sowie dessen wiederholte Aussage, dass die Zulässigkeit von Sperrklauseln nicht „ein für allemal“ beurteilt werden kann, erscheinen die Fünf-Prozent-Hürden auf Bundes- und Landesebene keineswegs in Stein gemeißelt. Eine neue Karlsruher Bewertung zumindest der Höhe der Sperrklausel könnte sich beispielsweise aus dem Ergebnis der jüngsten Bundestagswahl ergeben, bei der ein historischer Rekordwert von 15,7 Prozent der abgegebenen gültigen Zweitstimmen sperrklauselbedingt ohne Erfolgswert blieb (im Gegensatz zu 2009 mit 6,0 Prozent); hätte die Sperrklausel bei drei oder vier Prozent gelegen, wären FDP und AfD in den Bundestag eingezogen und nur 6,2 Prozent der Zweitstimmen unberücksichtigt geblieben. ${ }^{116}$ Angesichts dieser Entwicklung ist eine neue verfassungsgerichtliche Bewertung der Sperrklauseln für deutsche Parlamentswahlen - übrigens auch durch Landesverfassungsgerichte für die Landtagswahlen - keineswegs zuverlässig auszuschließen.

113 Vgl. Günter Bannas, Fünfprozenthürde ins Grundgesetz?, in: FAZ vom 11. März 2014, S. 1; Dieter Nohlen / Ulrich Battis, Pro und Contra: Die Fünf-Prozent-Hürde, in: bpb.de vom 10. Oktober 2013, http://www.bpb.de/dialog/wahlblog/170393/pro-und-contra-die-fuenf-prozenthuerde (Abruf jeweils am 31. März 2014). Dies würde allerdings eine weitergehende Ergänzung des GG erforderlich machen; denn bislang ist nicht einmal das Wahlsystem dort geregelt, was aber eine Vorbedingung für die Festlegung einer Sperrklausel wäre.

114 Vgl. BVerfGE, Bd. 129, S. 300, S. 335 f., Rn. 118.

115 Vgl. BVerfG, a.a.O. (Fn. 2), Rn. 72.

116 Vgl. Der Bundeswahlleiter, Endgültiges amtliches Ergebnis der Bundestagswahl 2013, Pressemitteilung vom 9. Oktober 2013, http://www.bundeswahlleiter.de/de/bundestagswahlen/BTW BUND_13/presse/w13034_Endgueltiges_amtliches_Ergebnis.html (Abruf am 31. März 2014). 